\title{
KINEMATICS AND MODELING OF THE INNER REGION OF M 83
}

\author{
Irapuan Rodrigues ${ }^{1,2}$, Horacio Dottori ${ }^{2,3}$, Rubén J. Díaz ${ }^{4,5}$, María P. Agüero ${ }^{5,6}$, And Damián Mast ${ }^{5,6}$ \\ ${ }^{1}$ IP\&D-Universidade do Vale do Paraíba - Av. Shishima Hifumi, 2911 - 12244-000 - São José dos Campos - SP - Brazil; irapuan@univap.br \\ ${ }^{2}$ Research Grant from CNPq, Brazil \\ ${ }^{3}$ Department of Astronomy, IF-UFRGS, CP 15051, CEP 91501-970, Porto Alegre, Brazil; dottori@if.ufrgs.br \\ ${ }^{4}$ Gemini Observatory, Southern Operations Center, La Serena, Chile; rdiaz@gemini.edu \\ ${ }^{5}$ Consejo Nacional de Investigaciones Científicas y Técnicas, Argentina \\ ${ }^{6}$ Observatorio Astronómico, Universidad Nacional de Córdoba, Argentina \\ Received 2007 February 24; accepted 2008 December 15; published 2009 March 26
}

\begin{abstract}
Two-dimensional kinematics of the central region of M 83 (NGC 5236) were obtained through three-dimensional NIR spectroscopy with the Gemini South telescope. The spatial region covered by the integral field unit $\left(\sim 5^{\prime \prime} \times 13^{\prime \prime}\right.$ or $\sim 90 \times 240 \mathrm{pc}$ ) was centered approximately at the center of the bulge isophotes and oriented southeast-northwest. The $\mathrm{Pa} \beta$ emission at half-arcsecond resolution clearly reveals spider-like diagrams around three centers, indicating the presence of extended masses, which we describe in terms of Satoh distributions. One of the mass concentrations is identified as the optical nucleus $(\mathrm{ON})$, another as the center of the bulge isophotes, similar to the $\mathrm{CO}$ kinematical center (KC), and the third as a condensation hidden at optical wavelengths (hidden nucleus, HN), coincident with the largest lobe in $10 \mu \mathrm{m}$ emission. We run numerical simulations that take into account $\mathrm{ON}, \mathrm{KC}$, and $\mathrm{HN}$ and four more clusters, representing the star-forming arc at the southwest of the optical nucleus. We show that $\mathrm{ON}, \mathrm{KC}$, and $\mathrm{HN}$ suffer strong evaporation and merge in 10-50 Myr. The star-forming arc is scattered in less than one orbital period, also falling into the center. Simulations also show that tidal striping boosts the external shell of the condensations to their escape velocity. This fact might lead to an overestimation of the mass of the condensations in kinematical observations with spatial resolution smaller than the condensations' apparent sizes. Additionally, the existence of two ILR resonances embracing the chain of $\mathrm{H}$ II regions, claimed by different authors, might not exist due to the similarity of the masses of the different components and the fast dynamical evolution of M 83 central 300 pc.
\end{abstract}

Key words: galaxies: individual (NGC 5236) - galaxies: kinematics and dynamics - galaxies: nuclei - galaxies: stellar content

Online-only material: color figures

\section{INTRODUCTION}

In spite of the peculiar structure of its circumnuclear region, NGC 5236 presents a well defined bulge that can be fitted by de Vaucouleurs' law in an annular region between radii $\approx 10^{\prime \prime}$ and $40^{\prime \prime}$ (Jensen et al. 1981; Gallais et al. 1991) corresponding to $\approx 180 \times 720 \mathrm{pc}$. The dramatic inward behavior begins approximately at a radius of $180-190 \mathrm{pc}$, where the main dust lanes associated with the galaxy bar spiral into a couple of $(\mathrm{J}-\mathrm{K})$ rings that, according to Elmegreen et al. (1998), might coincide with two inner Linblad resonances. These rings embrace an arc of about 20 star-forming condensations (Gallais et al. 1991; Heap et al. 1993; Harris et al. 2001) comparable to 30 Doradus in the Large Magellanic Cloud (Heap et al. 1993), clustered in four main knots (Elmegreen et al. 1998, Figure 4). The center of this arc is approximately $\approx 6.7$ to the southwest (SW) of the condensation known as the optical, or visible, nucleus $(\mathbf{O N})$ of NGC 5236. ON marks the center of the Elmegreen et al. (1998) inner nuclear ring, but not that of the outer nuclear ring, which is shifted 2".5 to the SW of the optical nucleus. $K$-band spectroscopy (Thatte et al. 2000) suggests a dynamical center coincident with the center of symmetry of the $K$-band external isophotes (KC), which is shifted $4^{\prime \prime}$ to the SW of the optical center (see Figure 3 in Mast et al. 2006; our Figure 1). This dynamical center is also suggested, although with smaller spatial resolution, by two-dimensional CO spectroscopy (Sakamoto et al. 2004). The region of Thatte's (2000) isophotal study coincides with that analyzed by Jensen et al. (1981), and is dominated by the bulge light.
The disk-like appearance of the CO kinematics interior to $300 \mathrm{pc}$ (Sakamoto et al. 2004) indicates that the bar perturbed disk survives well inside the galaxy bulge. How deep it extends into the nucleus is a question discussed in this paper. The presence of the hidden condensation (HN; Díaz et al. 2006a), which is larger and more massive than the optical nucleus, raises doubts about the true nature and the role of the optical nucleus. Furthermore, we also cast doubts on the existence of a double inner Lindblad resonance (ILR) embracing the arc of $\mathrm{H}$ II regions.

To better understand the richness of phenomena in the central region of NGC 5236 we performed three-dimensional near infrared spectroscopy at subarcsecond resolution using GEMINI+CIRPASS configuration (Díaz et al. 2006a). We observed a region including the $\mathrm{ON}, \mathrm{KC}$, and $\mathrm{HN}$. Our study is complemented by $N$-body simulations to test the stability of the M 83 central region, including the star-forming arc. In Section 2, we present our observations; in Section 3, we discuss the central kinematics and the three most important condensations, which present differentiated kinematics. Numerical simulations are presented in Section 4, and in Section 5 we present our conclusions.

\section{OBSERVATIONS}

We used the Cambridge IR Panoramic Survey Spectrograph (Parry et al. 2000, 2004) installed on the GEMINI South telescope in 2003 March. These observations, performed in queue mode, were taken with an integral field unit (IFU) 


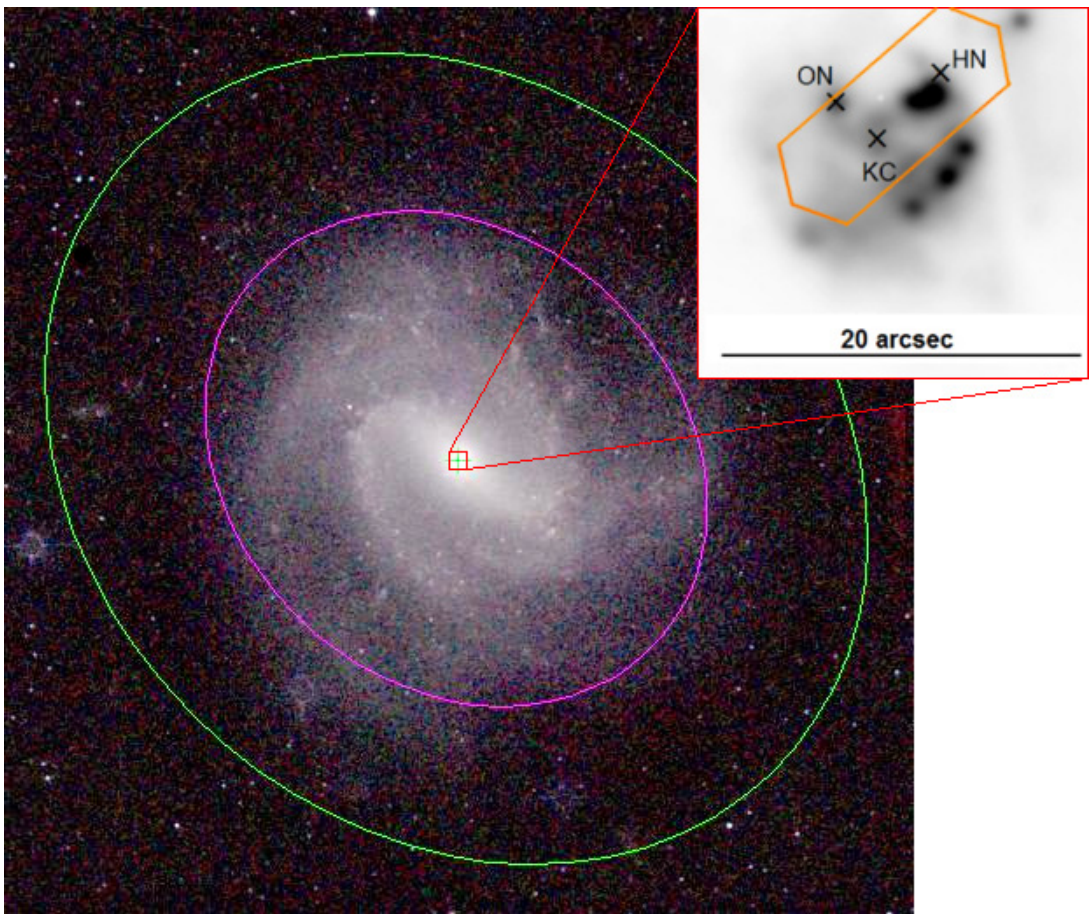

Figure 1. Two-mass JHK composite image showing two isophotes and a remarkable coincidence of their center with the bulge center KC. The blowup shows a GMOS/GEMINI-S co-added image of the central $20 \times 20 \operatorname{arcsec}^{2}$ with the position of KC, ON and HN. North is up and east is to the left. CIRPASS field true scale and orientation are outlined in orange.

(A color version of this figure is available in the online journal.)

sampling of $0^{\prime \prime} 36(6.4 \mathrm{pc})$ with an array with size $\approx 5^{\prime \prime} \times 13^{\prime \prime}$. The array of 490 hexagonal doublet lenses attached to fibers provides an area-filling factor of nearly $100 \%$. The IFU was oriented at P.A. $=120^{\circ}$ and centered slightly to the northwest (NW) of the kinematical center according to Mast et al. (2002) position. In Figure 1, a sketch of the detector position is shown. It was exposed for 45 minutes and covers a spectral range between $1.2 \mu \mathrm{m}$ and $1.4 \mu \mathrm{m}$, including $\mathrm{Pa} \beta 1.3 \mu \mathrm{m}$ and [Fe $\mathrm{II}] 1.26 \mu \mathrm{m}$, with a spectral resolution of approximately 3200 . The seeing was about 0 .'5; therefore, the focal plane was slightly subsampled by the configuration constraint.

The data were reduced using IRAF (distributed by the NOAO), ADHOC (two-dimensional kinematics analysis software developed by Marseille's Observatory) and SAO (spectra processing software developed by the Special Astrophysical Observatory, Russia). More details on the reduction are presented in Díaz et al. (2006b), and the general technique is thoroughly discussed by Díaz et al. (1999) and Mast et al. (2002).

\section{TWO-DIMENSIONAL KINEMATICS OF M 83 CENTRAL REGION AT LESS THAN 10 pc SPATIAL RESOLUTION}

In Figure 1, we present the composed JHK 2-mass image of M 83. The inset shows a blow-up of the central $400 \times 400 \mathrm{pc}^{2}$, where we detach the positions of $\mathrm{ON}, \mathrm{KC}$, and $\mathrm{HN}$. The astrometric positions of the condensations are given in Table 1 (the astrometry is discussed in Díaz et al. 2006b). In Figure 2, we show the brightness distribution at the $\mathrm{Pa} \beta$ continuum, the $\mathrm{Pa} \beta$ radial isovelocity contours, and the $\mathrm{Pa} \beta$ velocity dispersion map. The velocity dispersion is shown as the line FWHM (Table 2). In spite of the complex kinematics shown by the two-dimensional radial velocity map, we can distinguish ordered radial velocities in the form of spider diagrams around $\mathrm{ON}, \mathrm{KC}$, and $\mathrm{HN}$. As
Table 1

Condensation Data

\begin{tabular}{lccrcr}
\hline \hline Knot & $\begin{array}{c}\text { R.A. } \\
(\mathrm{J} 2000)\end{array}$ & $\begin{array}{c}\text { Decl. } \\
(\mathrm{J} 2000)\end{array}$ & $\begin{array}{c}R_{\max } \\
(\mathrm{pc})\end{array}$ & $\begin{array}{c}V_{\text {peak }} \\
\left(\mathrm{km} \mathrm{s}^{-1}\right)\end{array}$ & $\begin{array}{c}\sigma \\
\left(\mathrm{km} \mathrm{s}^{-1}\right)\end{array}$ \\
\hline KC & $13^{\mathrm{h}} 37^{\mathrm{m}} 0.56$ & $-29^{\circ} 51^{\prime} 56^{\prime \prime} .9$ & $65 \pm 5$ & $68 \pm 8$ & $82 \pm 10$ \\
ON & $13^{\mathrm{h}} 37^{\mathrm{m}} 0.98$ & $-29^{\circ} 51^{\prime} 55^{\prime \prime} .5$ & $8 \pm 1$ & $46 \pm 9$ & $110 \pm 10$ \\
$\mathrm{HN}$ & $13^{\mathrm{h}} 37^{\mathrm{m}} 0.46$ & $-29^{\circ} 51^{\prime} 53^{\prime \prime} .6$ & $45 \pm 8$ & $58 \pm 11$ & $96 \pm 10$
\end{tabular}

Notes. The $2 \sigma$ uncertainties in the astrometry of $\mathrm{ON}, \mathrm{KC}$, and $\mathrm{HN}$ are $0^{\prime \prime} .15$, $0^{\prime \prime} 8$, and 0.7 , respectively. The three last columns show characteristics of the disk around each one of the condensations: $R_{\max }$ is the largest extension along the line of the nodes where disk kinematics can be reliably distinguished. $V_{\text {peak }}$ is the peak velocity along the line of the nodes, corrected for inclination and $\sigma$ is the velocity dispersion at the position of the condensations.

is well known, a spider diagram indicates disk-like motions. The sizes of the disks around the three condensations, their maximum radial velocities along the line of the nodes, and their velocity dispersions at their centers of symmetry are presented in Table 1.

\subsection{The Ionized Gas Kinematics Around KC}

$\mathrm{KC}$ is the most extended of the three disks. Its center coincides, up to the margin of error, with the center of symmetry of the bulge isophotes (Jensen et al. 1981; Thatte et al. 2000), which in turn coincides with that of the $\mathrm{CO}$ kinematical center (Sakamoto et al. 2004). We recall that the GEMINI spatial resolution is at least 20 times higher than that of Sakamoto's $\mathrm{CO}$ observation.

The major axis of the spider diagram is at P.A. $=120^{\circ}$. From the spider diagram major-to-minor axis ratio, we derive an inclination of $68^{\circ} \pm 8^{\circ}$ for the gaseous disk. We have to be cautious at this point, because the minor axis velocity field seems 


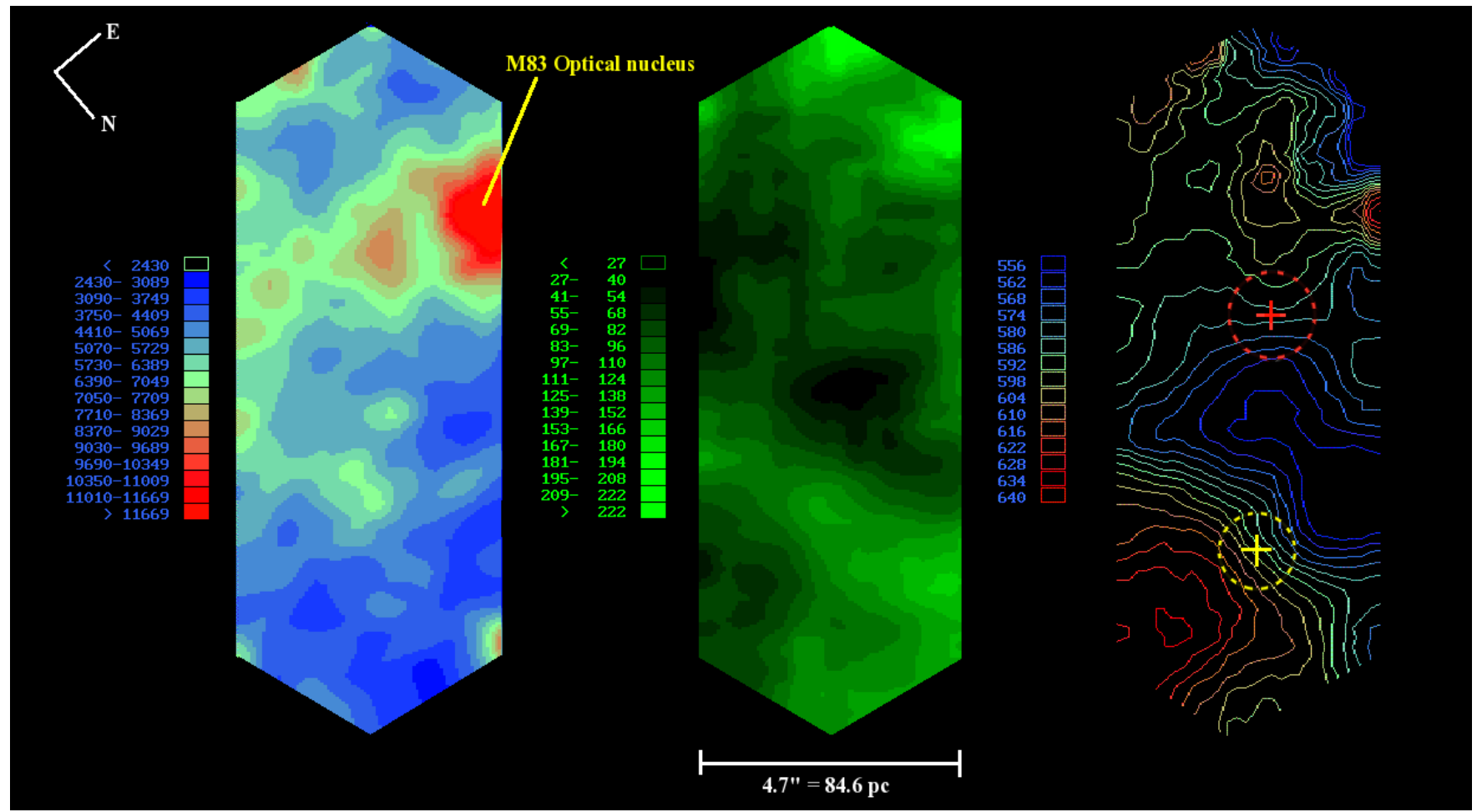

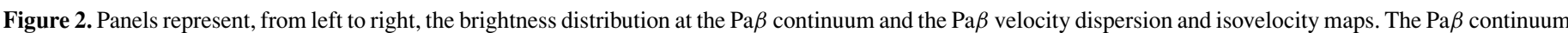

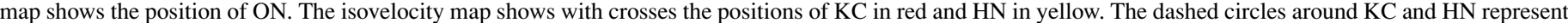
the astrometric uncertainty in their position.

to be perturbed. The same situation was faced by Sakamoto et al. (2004), who also quote two highly discrepant possible values for the inclination of the 300 pc CO disk. These authors finally chose the inclination angle of the large-scale galactic disk based on kinematical reasons. In our case, the best two-dimensional velocity model fitting to the observed velocity field is attained for an inclination angle of $25^{\circ} \pm 8^{\circ}$ (see Section 3.4), which coincides, up to the margin of error, with the small inclination of the large-scale M83 disk. A small angle also better fits the innermost scale rotation curves to Sakamoto's 300 pc disk one (see Figure 3). The inflow of matter along the bar into the central region might be mimicking the disks' high inclination. The $\mathrm{Pa} \beta$ disk around the KC and Sakamoto's CO disk present different orientations (see Figure 3 ), probably pointing to a phenomenon of transition between $x_{2}$ - and $x_{1}$-like orbits, as quoted by Sakamoto et al. (2004), or to a strong perturbation of the central structure, as discussed by Díaz et al. (2006a).

The spider diagram (see Figure 2) can be traced up to $R_{\max } \approx 65 \mathrm{pc}$, but it is strongly perturbed to the $\mathrm{E}$ at the receding extreme, and to the $\mathrm{W}$ at the approaching extreme. The $\mathrm{KC}$ rotation curve (Figure 4) was obtained from approximately 60 radial velocity points distributed along the whole disk. From this data set, we obtained 13 mean rotation velocities between 4 pc and $65 \mathrm{pc}$. Each velocity point entering the mean was weighted by $w(\alpha)=|\cos (\alpha)|$, where $\alpha$ is the position angle of the line joining the point to the disk center with respect to the line of the nodes. The disk around $\mathrm{KC}$ can be described in terms of a Satoh's-like spheroid with an effective radius of $R_{\mathrm{KC}}=38 \pm$ $8 \mathrm{pc}$ and a mass inside $R_{\max }$ of $M_{\mathrm{KC}} \approx(60.0 \pm 20) \times 10^{6} M_{\odot}$ (for a disk inclination of $25^{\circ}$ ). We note that an inclination of $68^{\circ}$ for the disk around $\mathrm{KC}$ would give a mass of $\approx(17 \pm 2) \times 10^{6} \mathrm{M}_{\odot}$, similar to that deduced spectroscopically by Thatte et al. (2000) from the ${ }^{12} \mathrm{CO}$ band-head velocity broadening.

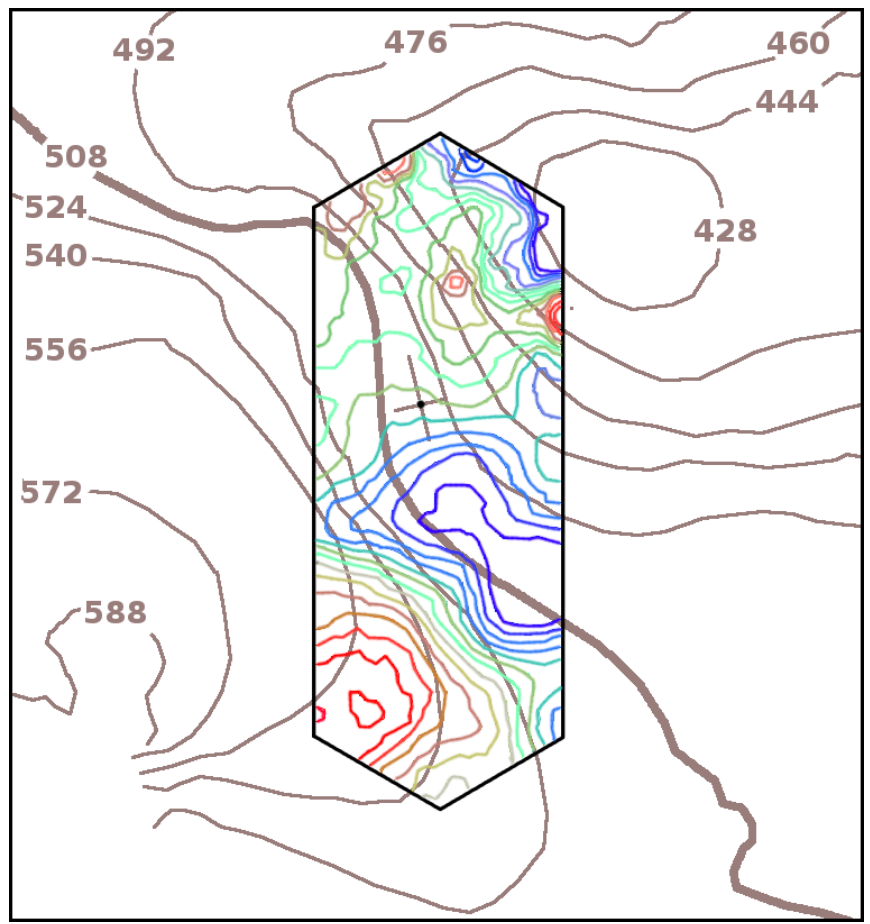

Figure 3. Central part of the Sakamoto et al. (2004) CO (2-1) velocity map (brown contours) superimposed on the $\mathrm{Pa} \beta$ isovelocity maps.

The velocity dispersion in the nonresolved central part is of the order of $80 \mathrm{~km} \mathrm{~s}^{-1}$ (see middle panel in Figure 2). Assuming that this velocity dispersion is due to a central mass concentration, namely a putative black hole $(\mathrm{BH})$, we determined the $\mathrm{BH}$ mass upper limit, $M_{\mathrm{BH}}$, by adding Keplerian 

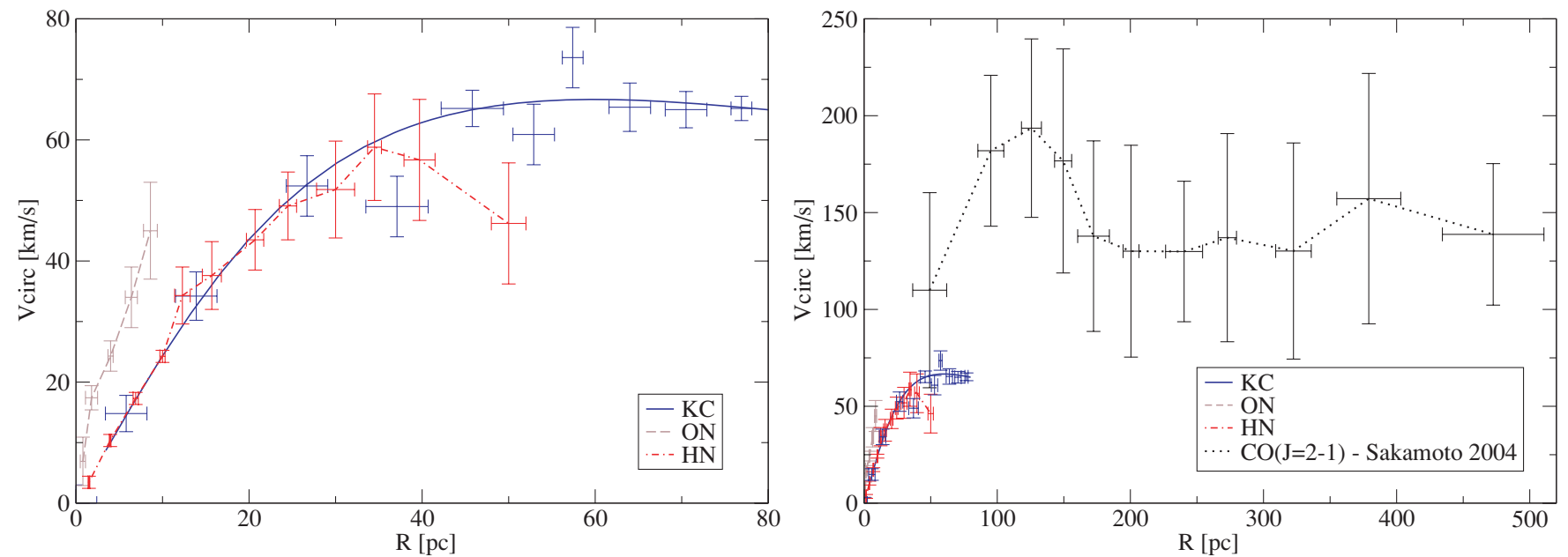

Figure 4. Rotation curves of the KC, the ON, and the HN. They are compared in the right panel to the $300 \mathrm{pc}$ CO disk rotation curve, as obtained from Sakamoto et al. (2004)

(A color version of this figure is available in the online journal.)
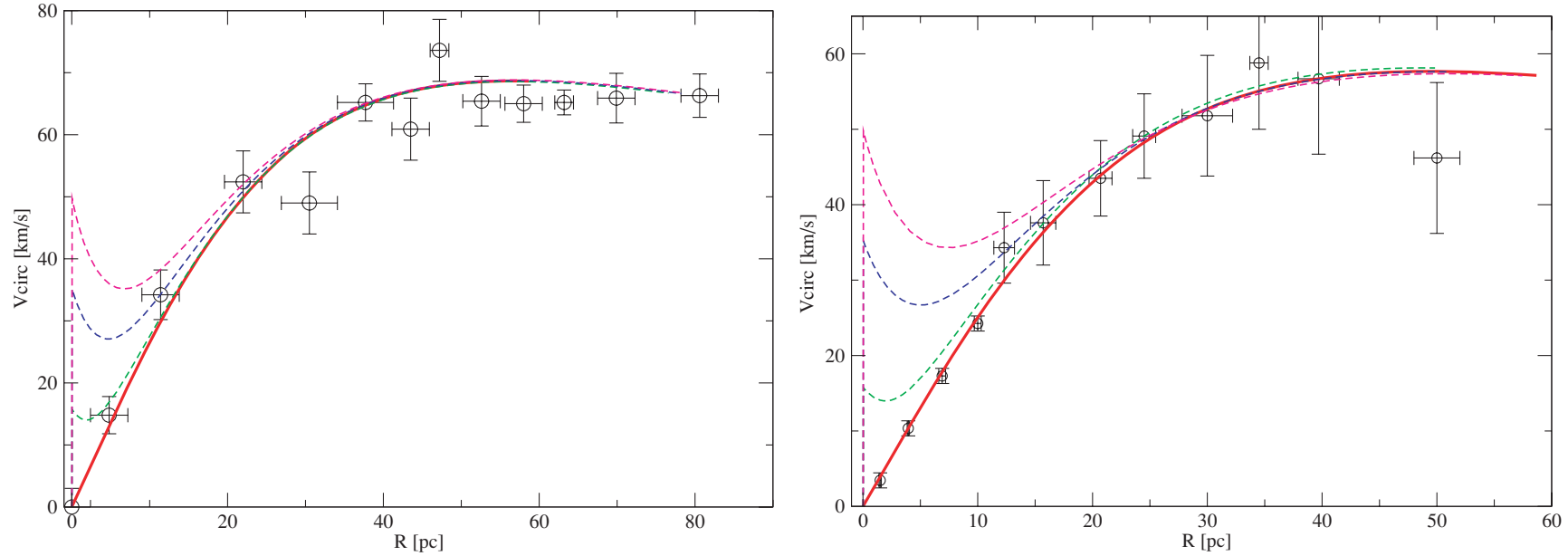

Figure 5. Rotation curves of $(a) \mathrm{KC}$ and $(b) \mathrm{HN}$ were fitted by Satoh's models (red line). The dashed lines (green, blue, and magenta in the electronic edition) were obtained by adding point-like sources of $M_{\mathrm{BH}}=0.2,1.0$, and $2.0 \times 10^{6} M_{\odot}$, convolved with a 9 pc Gaussian. $M_{\mathrm{BH}}=0.2 \times 10^{6} M_{\odot}$ is more compatible in both cases (A color version of this figure is available in the online journal.)

Table 2

Satoh Model-Fitting Parameters

\begin{tabular}{lccc}
\hline \hline Parameter & KC & ON & HN \\
\hline P.A. $\left({ }^{\circ}\right)$ & 120 & 120 & 64 \\
i $\left(^{\circ}\right)$ & $25.0 \pm 8$ & $60 \pm 10$ & $62 \pm 8$ \\
$R_{\text {eff }}(\mathrm{pc})$ & $38.0 \pm 4$ & $8 \pm 1$ & $33 \pm 3$ \\
$M_{K}\left(10^{6} M_{\odot}\right)$ & $60.0 \pm 20$ & $4 \pm 2$ & $20 \pm 7$ \\
$M_{B H}\left(10^{6} M_{\odot}\right)$ & $0.2-1.0$ & $<1.0$ & $0.2-1.0$ \\
\hline
\end{tabular}

rotation curves convolved with a 9 pc Gaussian to that of the Satoh disk, and constraining the resulting Satoh+Kepler rotation curve with the central velocity dispersion and the errors of the measured velocity points. The result of this procedure is shown in Figure 5 for $M_{\mathrm{BH}}=0.2,1.0$, and $2.0 \times 10^{6} M_{\odot}$. The best fit is quoted in Table 2.

This analysis points to $\mathrm{KC}$ as the true nucleus of $\mathrm{M} 83$.

\subsection{The Ionized Gas Kinematics Around HN}

Located at $R=7 " .8 \pm 0.7$ to the NW of KC (Figure 2), the spider diagram of the ionized gas around $\mathrm{HN}$ presents a P.A. $=64^{\circ}$. The form of the spider diagram (open legs) points to a more concentrated and more homogeneous distribution than that of the KC. It is probably a cannibalized dwarf satellite of M 83, as discussed by Díaz et al. (2006b). To derive the rotation curve as for $\mathrm{KC}$, we used a distribution of more than 50 points along the whole disk, which provided 13 mean rotation velocities up to $R=50 \mathrm{pc}$. In Tables 1 and 2 , we present the kinematical data for $\mathrm{HN}$ and the derived Satoh disk parameters. The mass of $\mathrm{HN}$ was also derived from the rotation curve, as well as that of the $\mathrm{BH}$ candidate at its center (see Table 2), through the velocity dispersion, following the procedure previously outlined for $\mathrm{KC}$.

\subsection{The Ionized Gas Kinematics Around ON}

ON is traditionally referred to as the off-centered optical nucleus of M83. Sakamoto et al. (2004) proposed that it could be a cannibalized satellite.

From photometric data, Elmegreen et al. (1998) derived a mass of $4 \times 10^{6} M_{\odot}$ and Thatte et al. (2000) of $2.5 \times 10^{6} M_{\odot}$. From ${ }^{12} \mathrm{CO}$ band-head $2.293 \mu \mathrm{m}$ velocity dispersion, Thatte et al. (2000) derived a kinematical mass of $1.3 \times 10^{7} M_{\odot}$ within $5.4 \mathrm{pc}$, assuming that the optical nucleus is virialized. The mass of ON derived photometrically disagrees by a factor of 
(a)

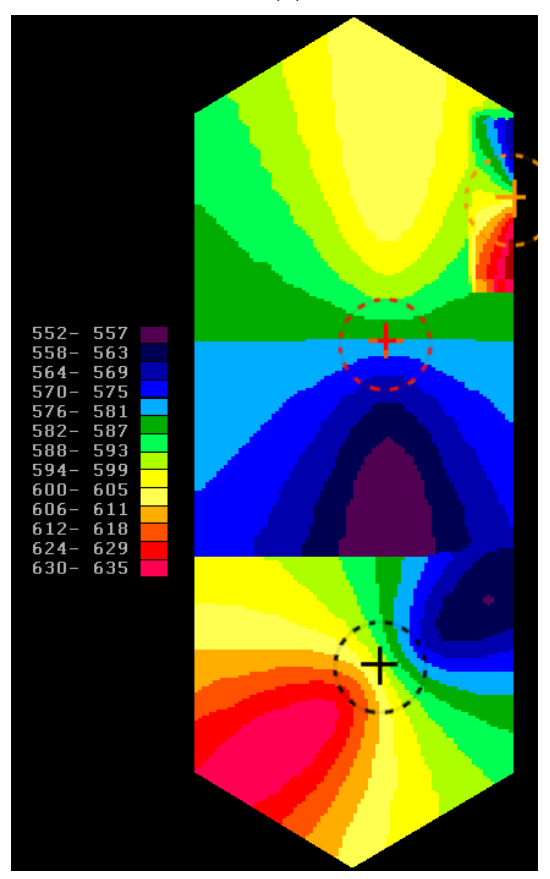

(b)

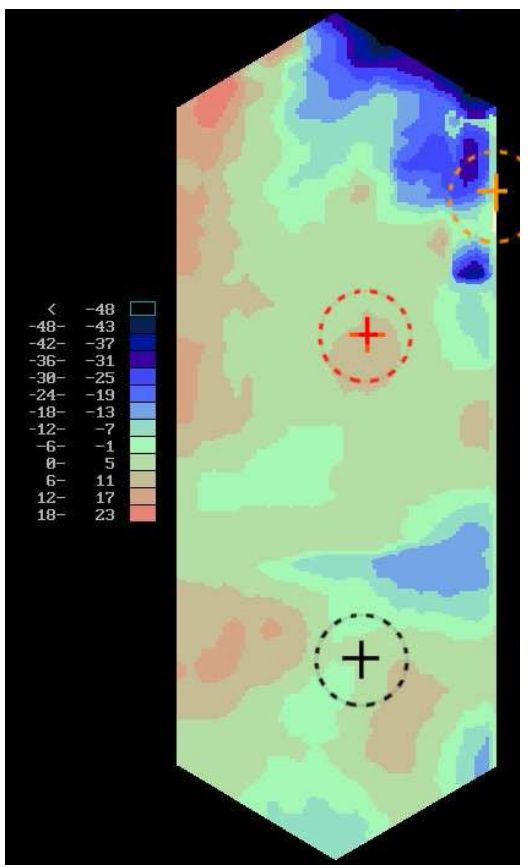

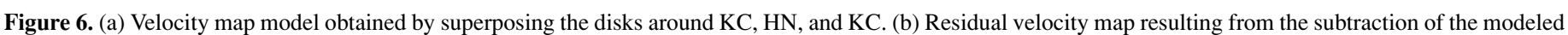
velocity map from the observed one.

4-5 from that derived spectroscopically, probably indicating a system with a nonvirialized periphery, as discussed in Section 4 in connection with the many-body interaction. The disk around $\mathrm{ON}$ is the smallest of the three disks discussed in this paper. The ON kinematical center is slightly shifted to the SW of the ON continuum peak at $1.3 \mu \mathrm{m}$, as can be seen in Figure 2 . The velocity gradient across $\mathrm{ON}$ is determined from data near the spatial resolution limit. We are probably dealing with problems of oversampling. The rotation curve with mean errors is shown in Figure 4. The mass of ON was also derived from the rotation curve, as well as that of the putative $\mathrm{BH}$ at its center (see Table 2), using the velocity dispersion and the procedure previously outlined for KC. Our determination of the mass agrees very well with that obtained from photometric data.

\subsection{Fitting the Whole Central Radial Velocity Field of the Gas}

The data in Tables 1 and 2 allow us to generate a composed radial velocity map model (Figure 6a) to be compared with the observed one. The fitting of $\mathrm{KC}$ was the most critical due to the difficulty in the determination of its disk inclination angle (3.1). Several fits were made, keeping constant the inclination angles of the disks around $\mathrm{ON}$ and $\mathrm{HN}$ and allowing the one associated with $\mathrm{KC}$ to vary from $25^{\circ}$ to $60^{\circ}$. We verified that the disk-like residual around $\mathrm{KC}$ decreases dramatically when approaching smaller inclinations. Finally, the smallest residuals are obtained for an inclination of $25^{\circ} \pm 5^{\circ}$. The final map is shown in Figure 6(b). This inclination is in good agreement with the inclination of the large-scale M83 disk and that of the inner 300 pc, derived from CO kinematics (Sakamoto et al. 2004), as can be seen in Figure 4. This again points to a continuity of the galactic disk kinematics from a kiloparsec scale to the smaller scales studied in this paper, although variations in the position angles of the lines of nodes are observed at $300 \mathrm{pc}$ and at scales smaller than $100 \mathrm{pc}$ as well (see Figure 3).

Figure 6(b) shows that around $\mathrm{KC}, \mathrm{ON}$, and $\mathrm{HN}$ the residuals are mainly at the noise level, indicating that the interpretation of the observed velocity map as resulting from the superposition of three disks is, from a qualitative point of view, essentially correct. Nevertheless, deviations from rotation points to a more complex situation and indicates that the mass estimations based on rotation curves are barely approximate. As an example we detect anomalous blueshifted kinematics at the east extreme (upper right) above $\mathrm{ON}$, and redshifted kinematics to the west of HN. These regions coincide with the high-velocity dispersion zones in Figure 2. These might indicate regions of inflow of gas along the bar falling into the central region.

The fit of the rotation curve, from the scale of tens to hundreds of parsecs (Figure 3), does not seem to confirm the existence of double ILR, as claimed by Elmegreen et al. (1998) and Sakamoto et al. (2004).

\section{4. $N$-BODY SIMULATIONS}

In order to understand the dynamical evolution of the M 83 central region, we performed $N$-body simulations of a system composed of $\mathrm{KC}, \mathrm{HN}, \mathrm{ON}$ and four condensations representing the star-forming arc. Hernquist's models were used for $\mathrm{KC}$, HN, and ON (Hernquist 1993). We included stellar and gaseous components. The models were constrained by the bodies' rotation curves. Figure 7 shows the model rotation curves adjusted in order to fit the observational data. The model parameters are quoted in Table 3. The four condensations on the star-forming arc were represented by Plummer's models, with 1000 particles each, with positions and masses corresponding to regions 2-5 in Figure 8 of Elmegreen et al. (1998), where region 2 has $M_{2}=1.3 \times 10^{6} M_{\odot}$, regions 3 and 4 have $M_{3}=$ $M_{4}=1.4 \times 10^{6} M_{\odot}$, and region 5 has $M_{5}=1.8 \times 10^{6} M_{\odot}$. All Plummer models have a core radius of $1.5 \mathrm{pc}$ and a cutoff radius of $15 \mathrm{pc}$.

A large set of simulations was run in order to refine the models and orbital parameters used in the final simulation. The orbital parameters were chosen considering that M83 rotates clockwise. Inclinations and position angles are as in Table 2. 

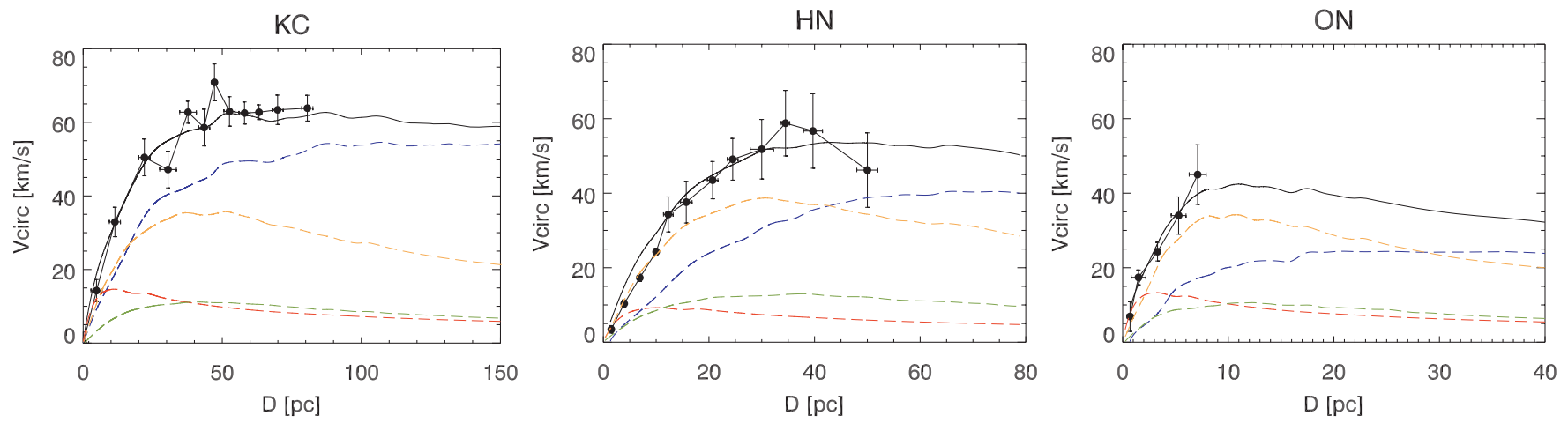

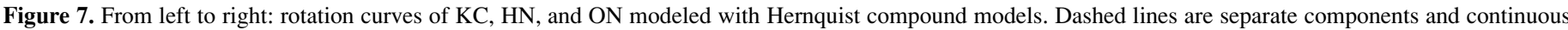
line is the model circular speed. Black dots with error bars are from CIRPASS data.

(A color version of this figure is available in the online journal.)

Table 3

$\mathrm{KC}, \mathrm{HN}$, and ON Model Parameters

\begin{tabular}{lccc}
\hline \hline Parameter & KC Model & HN Model & ON Model \\
\hline Number of particles in disk & 16384 & 8192 & 4096 \\
Disk mass & 15.0 & 13.0 & 3.5 \\
Disk radial scale length & 17.0 & 13.0 & 4.5 \\
Disk vertical scale thickness & 1.7 & 0.8 & 0.4 \\
Reference radius $R_{\text {ref }}$ & 42.5 & 30 & 8.0 \\
Toomre Q at $R_{\text {ref }}$ & 1.5 & 1.5 & 1.5 \\
\hline Number of particles in gas disk & 16384 & 8192 & 4096 \\
Gas disk mass & 1.5 & 1.5 & 0.35 \\
Gas disk radial scale length & 17.0 & 13.0 & 4.5 \\
Gas disk vertical scale thickness & 1.7 & 0.8 & 0.4 \\
Toomre Q at $R_{\text {ref }}$ & 1.5 & 1.5 & 1.5 \\
\hline Number of particles in bulge & 512 & 512 & 512 \\
Bulge mass & 1.3 & 0.5 & 0.3 \\
Bulge radial scale length & 3.4 & 4.0 & 1.0 \\
\hline Number of particles in spherical component & 16384 & 8192 & 4096 \\
Halo mass & 150.0 & 40.0 & 7.0 \\
Halo cutoff radius & 200.0 & 80.0 & 45.0 \\
Halo core radius & 17.0 & 25 & 4.5 \\
\hline
\end{tabular}

Notes. Simulations were done in a system of units with $G=1$. Model units scale to physical ones such that: length unit is $1 \mathrm{pc}$, velocity unit is $65.58 \mathrm{~km} \mathrm{~s}^{-1}$, mass unit is $1 \times 10^{6} M_{\odot}$, and time unit is $14909.92 \mathrm{yr}$.

Here, we suppose that clusters $2-5$ on the star-forming arc, as well as ON are in circular clockwise (prograde) orbits in the plane of the external disk of M83. According to Díaz et al. (2006a), HN is the remnant of a captured galaxy that triggered the star formation along the arc of $\mathrm{H}$ II regions. The form of the arc and its position with respect to HN lead us to adopt a circular counter-clockwise orbit for HN.

The simulations were performed with Gadget2 (Springel 2005). A total of 91,552 particles were used, and the simulations were run for $300 \mathrm{Myr}$, starting at the present time configuration. We considered starting at a previous epoch, before the formation of the arc of $\mathrm{H}$ II regions, but the complexity of the environment at the M83 central region and the number of parameters required to qualitatively take into account all possible configurations in the past would be a matter for a paper especially devoted to that study.

The simulation (see Figure 8) shows that the galaxy nucleus $(\mathrm{KC})$, the optical nucleus (ON), and the hidden nucleus (HN) would form a single massive core in about $16 \mathrm{Myr}$. All Plummer models (clusters 2-5) fall into the nucleus in $130 \mathrm{Myr}$.

Considering the range of uncertainties in the orbit determination, we can state that this massive core would finally settle as the new nucleus of M 83 in a few tens of Myr, implying a net growth of the central galactic mass. Furthermore, the whole star formation and nuclei merging event would last about the time of a global galactic revolution (about $150 \mathrm{Myr}$ at the radius of $5 \mathrm{kpc})$.

Simulations also show that tidal striping of the condensations boosts the velocity field of the external shell to escape velocity, which, if mistaken for the dispersion velocity of systems in equilibrium, might lead to an overestimation of their masses. This seems to be the case for the disagreement between the photometric and kinematic masses derived by Thatte et al. (2000) for ON.

The circumnuclear $\mathrm{H}$ II regions arc is far from being a stable system. In fact, it will spread out in an orbital time and be swallowed by the new refurbished nucleus in a period slightly longer than the time of merging of $\mathrm{ON}, \mathrm{KC}$, and $\mathrm{HN}$. Therefore, the claimed existence of two ILRs is difficult to sustain.

\section{CONCLUSION}

We performed CIRPASS two-dimensional spectroscopy at $\mathrm{Pa} \beta$ and its continuum with the GEMINI-S telescope. $\mathrm{Pa} \beta$ 

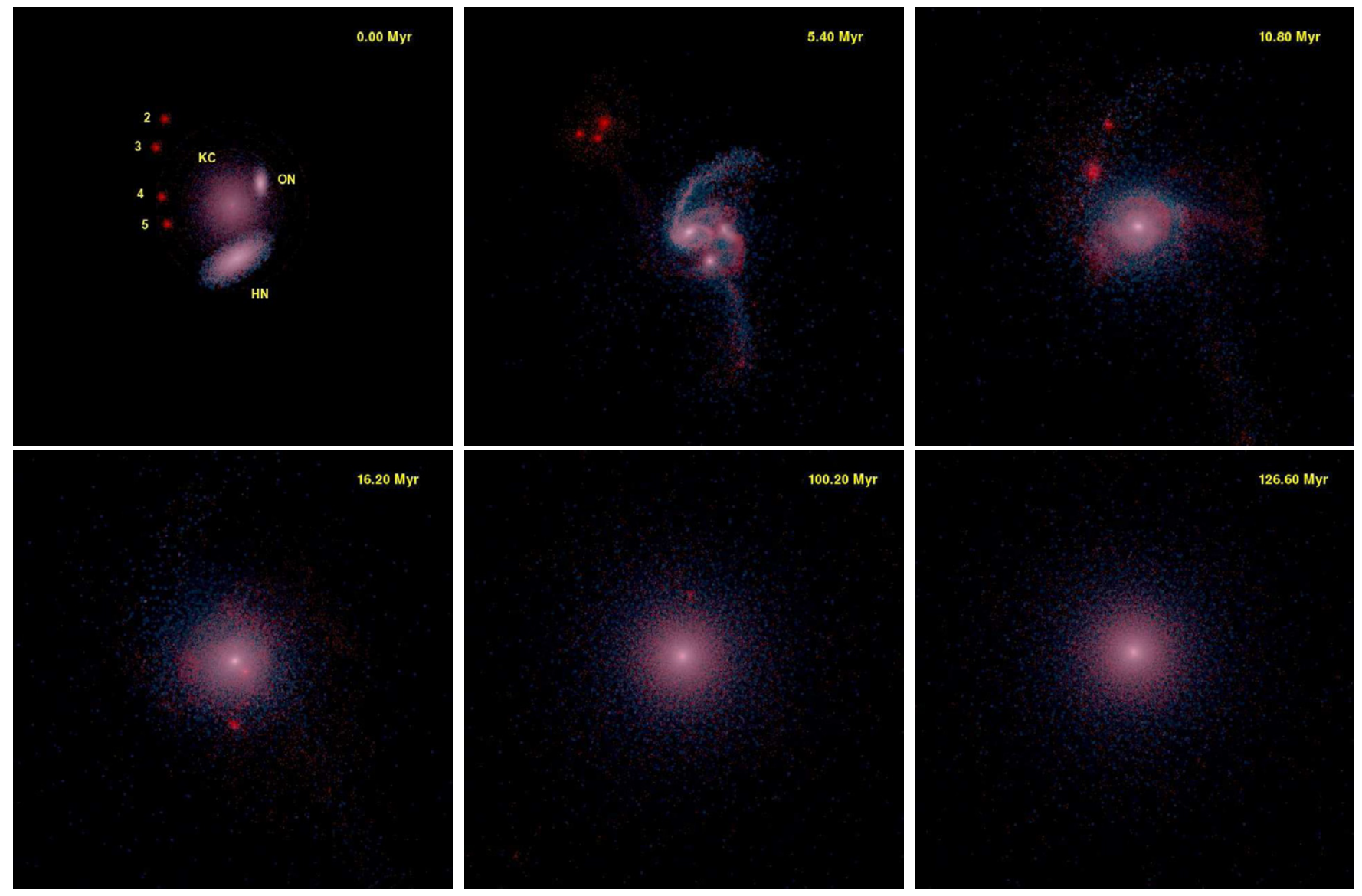

Figure 8. Evolution of the M83 central region model. ON and the Plummer models (2-5) are in the prograde (clockwise) orbits around KC, while HN is in a retrograde orbit. KC, HN, and ON merge in 16 Myr. The last Plummer sphere (cluster 4) coalesces with the central body in 130 Myr. Gas particles are blue; stellar particles are red. Spherical components of $\mathrm{KC}, \mathrm{HN}$, and $\mathrm{ON}$ models are not shown for clarity.

(A color version of this figure is available in the online journal.)

spectroscopy allows for penetration of the dust in the direction of $\mathrm{KC}$ and $\mathrm{HN}$, and for determination of the ionized gas kinematics in the central $\approx 5^{\prime \prime} \times 13^{\prime \prime}$ embracing $\mathrm{ON}, \mathrm{KC}$, and $\mathrm{HN}$. The kinematics can mainly be explained in terms of three disks around each one of the condensations, KC, HN, and ON. Perturbations are visible when the three-disk model is subtracted from the observations. The line of the nodes of a tens of parsecs-scale disk around $\mathrm{KC}$ is rotated with respect to that of the hundreds of parsecs-scale $\mathrm{CO}$ one, which in turn is rotated with regard to the line of the nodes of the outer galactic disk. They are most probably co-planar up to the margin of error. Nevertheless, a relative warp of the galactic disk inward of up to $40^{\circ}$ cannot be ruled out.

Besides the mass of the condensations $\mathrm{KC}, \mathrm{HN}$, and $\mathrm{ON}$, we inferred upper mass limits for the putative BHs that could be associated with them.

Our simulation shows that the three nuclei, $\mathrm{KC}, \mathrm{HN}$, and ON, will merge in a few tens of Myr. The disruption of the circumnuclear $\mathrm{H}$ II regions arc in an orbital time shows that even if ILR do exist, this is not enough to ensure the stability of the H II regions' orbits.

We are witnessing a dramatic change in the central region of M83, the nearest galaxy with strong nuclear star formation, which may in a few Myr change the mass at its kinematical center by a factor of 2 , as well as the global aspect at a hundred parsec scale. Although HN was interpreted as a captured dwarf satellite, the metamorphosis of the M 83 central region is independent of the origin of $\mathrm{HN}$.

I.R. and H.D. acknowledge support from CNPq (Brazil). I.R. also acknowledges support from FAPESP (Brazil). R.D., M.A. and D.M. acknowledge the support of CONICET grant PIP 5697. We acknowledge support from Secyt-Capes Agreement, project 035/07. We acknowledge the Instituto Nacional de Pesquisas Espaciais (INPE/MCT, Brazil) for providing computer time for part of the simulations. Some simulations were also run at IF-UFRGS, Brazil. The Gemini Observatory is operated by the Association of Universities for Research in Astronomy, Inc., under a cooperative agreement with the NSF on behalf of the Gemini partnership: NSF (USA), STFC (United Kingdom), NRC (Canada), ARC (Australia), MINCYT (Argentina), CNPq (Brazil) and CONICYT (Chile). This publication makes use of data products from the 2MASS (Two Micron All Sky Survey), which is a joint project of the University of Massachusetts and the Infrared Processing and Analysis Center/California Institute of Technology, funded by the NASA and the NSF.

\section{REFERENCES}

Díaz, R., Carranza, G., Dottori, H., \& Goldes, G. 1999, ApJ, 512, 623 Díaz, R. J., Dottori, H., Aguero, M. P., Mediavilla, E., Rodrigues, I., \& Mast, D. 2006a, ApJ, 652, 1122 
Díaz, R. J., Dottori, H., Mediavilla, E., Aguero, M., \& Mast, D. 2006b, New Astron. Rev., 49, 547

Elmegreen, D. M., Chromey, F. R., \& Warren, A. R. 1998, AJ, 116, 2834

Gallais, P., Rouan, D., Lacombe, F., Tiphene, D., \& Vauglin, I. 1991, A\&A, 243, 309

Harris, J., Calzetti, D., Gallagher, J. S., Conselice, III, C. J., \& Smith, D. A. 2001, AJ, 122, 3046

Heap, S. R., Holbrook, J., Malumuth, E., Shore, S., \& Waller, W. 1993, BAAS 25,840

Hernquist, L. 1993, ApJS, 86, 389

Jensen, E. B., Talbot, R. J., \& Dufour, Jr, R. J. 1981, ApJ, 243, 716

Mast, D., Díaz, R. J., \& Agüero, M. P. 2006, AJ, 131, 1394
Mast, D., Díaz, R. J., Agüero, M. P., Weidmann, W., Carranza, G., \& Gimeno, G. 2002, Bol. Asoc. Argen. Astron., 45, 74

Parry, I., et al. 2004, in Proc. SPIE 5492, CIRPASS: Description, Performance, and Astronomical Results, Ground-based Instrumentation for Astronomy, ed. A. F. M. Moorwood \& M. Iye (Bellingham, WA: SPIE), 1135

Parry, I. R., et al. 2000, in Proc. SPIE 4008, CIRPASS: An NIR Integral Field and Multi-object Spectrograph, Optical and IR Telescope Instrumentation and Detectors, ed. M. Iye \& A. F. Moorwood (Bellingham, WA: SPIE), 1193

Sakamoto, K., Matsushita, S., Peck, A. B., Wiedner, M. C., \& Iono, D. 2004, ApJ, 616, L59

Springel, V. 2005, MNRAS, 364, 1105

Thatte, N., Tecza, M., \& Genzel, R. 2000, A\&A, 364, L47 